\title{
Multicriteria Decision Support Would Avoid Overdiagnosis and Overtreatment
}

\author{
Vije Kumar RAJPUT ${ }^{\mathrm{a}}$, Jack DOWIE ${ }^{\mathrm{b}, \mathrm{c}, 1}$ \\ and Mette Kjer KALTOFT ${ }^{\mathrm{c}}$ \\ ${ }^{a}$ Stonydelph Health Centre, Tamworth, UK \\ ${ }^{b}$ London School of Hygiene and Tropical Medicine \\ ${ }^{c}$ University of Southern Denmark
}

\begin{abstract}
Population-level studies confirm the existence of significant rates of overdiagnosis and overtreatment in a number of conditions, particularly those for which the screening of asymptomatic individuals is routine. The implication is that the possibility of being overdiagnosed and/or overtreated must be mentioned as a possible harm in generating informed consent and participation from the individual invited to be screened. But how should the rates of such preference-insensitive population-level phenomena be introduced into preference-sensitive individual decision making? Three possible strategies are rejected, including the currently dominant one that involves presenting the rates relevant to overdiagnosis and overtreatment as discrete pieces of information about a single criterion (typically condition-specific mortality). Extensive quotation from a review of cancer decision aids confirms that processing this complex and isolated information is not a practical approach. However, the task is unnecessary, since an outcome-focused multicriteria decision support tool will incorporate the effects of overdiagnosis and overtreatment - along with the effects of any underdiagnosis and undertreatment.
\end{abstract}

Keywords: Overdiagnosis, overtreatment, multi-criteria decision support

\section{Introduction}

There has been growing recognition of the possibility, indeed likelihood, that healthcare delivery is characterised by phenomena variously labelled Over-Detection/ OverTesting/Over-Diagnosis (OD), with resulting Over-Treatment (OT), increasingly referred to as 'too much medicine'. The possibility of the obverse Under-phenomena (UD, UT) is well-recognised, but not currently regarded with such concern, and, interestingly, by implication, seen as a separable issue.

Interest in Decision Support Tools (DSTs) has simultaneously expanded in recent years, largely as a result of the growing commitment to 'shared decision making' by healthcare professionals and the consequent need for more effective and detailed communication with autonomous patients regarding the decision being made. Legal changes in relation to the obtaining of informed and preference-based consent have been another stimulus and this is one likely to become more important in the increasingly digital age that patients inhabit.

${ }^{1}$ Corresponding author, Jack Dowie, LSHTM, 15-17 Tavistock Place, London, UK WC1H 9SH; E-mail: jack.dowie@1shtm.ac.uk 
These two trends are related, but not easily integrated. Clinical decision making, whether shared or not, takes place at the individual level ex ante the rest of their life. In contrast, $\mathrm{OD} / \mathrm{OT}$ are group/population level constructs that can only be measured at that level and ex post - as the percentage of those who had a disease or condition detected, diagnosed, and treated, from which they would not have died or experienced lifeaffecting symptoms. The earlier detection and diagnosis may well have been correct, in the sense that a tumour was present in the individual and was correctly identified as 'cancer' according to standard definitions. But it would have - with probability OD/OT - remained 'indolent/benign' and not affected the individual's length of life or health.

Whether the overdiagnosis rate established by follow-up studies at a population level is $22 \%$, one finding for breast cancer screening, or $42 \%$, the parallel finding for prostate cancer screening [1], the question arises: how should the phenomenon, and its extent, be introduced into a clinical process committed to making decisions for which preferencesensitive informed consent has to be obtained? The question is relevant to the 'empowered physician' of futurist Bertalan Mesko [2], as well as to the digitallyempowered citizen with whom that future physician will be engaging. Brodersen [3] has rightly emphasised the conceptual complexity of the issue and communication task:

"All health professionals, politicians, health authorities, patients, and citizens, in general, have a stake in the answer to the question: what is the risk of being overdiagnosed? However, to answer this question, the denominator or the comparator must be defined. The risk of being overdiagnosed in cancer screening could be split into numerous questions, for example, (1) how many in a cohort invited to screening are overdiagnosed with cancer? (2) How many of the screening participants are overdiagnosed with cancer? (3) How many of the screening-detected cancers are overdiagnosed? (4) How many deaths from cancer are prevented compared to how many screening participants are overdiagnosed with cancer? "[3] (p81)

Housten and colleagues have recently reviewed how OD/OT is treated - or not - in 85 cancer screening patient decision aids [4]. Their systematic review embraces the various verbalisations of the concept/s which avoid using the specific OD/OT terms, such as 'experiencing testing or treatment which would turn out to have been unnecessary'. They emphasise the need for improved understanding of the phenomena at both individual and collective levels, especially via decision support. "The trade-offs regarding cancer screening and how to communicate them persist and warrant the development of effective communication strategies to support decision making. Moreover, there is a strong ethical need to include the potential harms of cancer screening, including overdiagnosis, that can be understood by a broad population."'[4] (p9).

\section{Method}

How should overdiagnosis and overtreating be dealt with in point of care decision making, where the clinical task is to identify the preference-sensitive optimal option for the person? From the Housten systematic review we identified four possible strategies for introducing OD/OT considerations into an individual decision process, within or outside a clinical consultation. The four strategies uncovered were then assessed in terms of their communication complexity and ability to meet the requirements of informed and preference-based consent. 


\section{Result}

\subsection{Strategy 1}

Inform the person that there is a possibility of OD/OT, perhaps with some qualitative verbal quantification ('small chance', 'moderate risk'), but no numerical rate/s, even in the form of uncertainty ranges.

This strategy fails to meet the minimum requirements for informed consent, let alone preference-based informed consent. It is often adopted where the aim is to increase the uptake of an intervention believed to be in the interests of the patient.

\subsection{Strategy 2}

Provide relevant numerical rate/s of OD/OT as discrete information to be input into a verbal deliberative process, either without a decision aid or incorporated within one.

This is the current dominant strategy, so we quote extensively from four of the 67 breast cancer screening aids that introduced OD/OT according to the Housten systematic review [4]. (The remaining 18 aids did not mention this possibility.)

- $\quad$ 'Breast screening' leaflet

[Available from: https://patient.info/cancer/breast-cancer-leaflet/breastscreening]

"An independent review in the UK in 2012 concluded that breast screening does save lives. If 10,000 women are screened from when they are 50 to when they are 70 , around 43 deaths would be prevented... it was concluded that for the 10,000 women screened from when they are 50 to 70,129 women would be over-diagnosed. The Cochrane review found that for the 2,000 women screened over 10 years, 10 women would have unnecessary treatment. In this analysis, for every life saved, ten women would have treatment which was not necessary. In the UK, the NHS screening programme estimates that for every life saved, three women have treatment that they didn't need."

- 'It's your Choice' [Available from: https://bit.ly/SydneyBCAid]

"Out of 1000 women who have breast screening for 25 years: 5 women avoid dying from breast cancer because of screening and 14 women still die from breast cancer; 103 women are diagnosed with breast cancer. Of these, 30 women experience over-detection: they are diagnosed and treated for a cancer that would not have caused any trouble and 73 women are diagnosed with breast cancer that is not over-detection....More women experience over-detection than avoid dying from breast cancer." (For a report on the trial of this aid see [5]).

- Health Decision [Available from:

https://www.healthdecision.org/tool\#/tool/mammo]

"Studies show that $10-30 \%$ of tumors found on a screening mammogram will not grow or spread fast enough to affect a woman's life. Case example: Patient is 50 years old; no family history of breast cancer; no previous breast biopsy; 
breast density is "unknown"; race/ethnicity White. For 1000 such women aged 50 for 10 years No mammogram: 29 are diagnosed with BC; 971 are not diagnosed with and will not have $\mathrm{BC} ; 24$ survive $\mathrm{BC}$ with or without screening. Biennial mammogram: 33 are diagnosed with $\mathrm{BC}$ : 24 survive $\mathrm{BC}$ with or without screening, 1 saved from a $\mathrm{BC}$ death, 4 die from $\mathrm{BC}, 4$ extra are overdiagnosed from screening. 967 are not diagnosed with BC: 587 no BC, recalls or biopsies, 380 recalled for one or more false alarms, 63 undergo a biopsy which is normal."

- 'Is a mammogram right for me' Canadian Cancer Society [Available from: http://www.mybreastsmytest.ca/en/]

"In Canada, about 1 out of 215 women aged 50-69 who go for a mammogram as part of a provincial screening program will be diagnosed with breast cancer. Of the 215, 199 will get the 'all clear', 16 are called for more tests, 15 get the 'all clear', 1 will have breast cancer... For every breast cancer found, approximately $1-10 \%$ are non-life threatening."

These examples confirm that valiant attempts to communicate about OD/OT, even when accompanied by pictograms, are likely to result in confusion, misinterpretation, or simple abandonment of any attempt to absorb. Our cognitive competencies are not up to the task of dealing with the complex results produced, however attractively communicated, and certainly not in the time likely to be allocated at the point of decision. In our view the task is not one that can be addressed without support which embeds the relevant numbers in a decision framework. Simply 'being informed' about them without knowing how to process them in decision making is of dubious value.

\subsection{Strategy 3}

Include 'Being OD/OT' (or something similar) as a separate criterion in a Multi-Criteria Decision Analysis (MCDA)-based decision support tool (DST).

In this sort of decision support tool the criterion 'avoiding being OD/OT' would be assigned a rate from the literature. The individual concerned would then assign a weight to 'being OD/OT' relative to the other criteria in the tool, such as the length and quality of life and treatment burden. However, as soon as this strategy is spelled out, it is clear that this is not a valid one, since it will be the consequences of OD/OT not being OD/OT which is of concern. These consequences will be incorporated in the other criteria and. while there may be annoyance attached to simply being OD/OT, we assume the weight attached to this, as opposed to the consequences, will be negligible.

\subsection{Strategy 4}


This strategy emerges as dominant for three reasons. One, entering in the DST the best individualised performance rates of options on the key outcome criteria - all-cause and condition-specific mortality, and various forms of all-cause and condition-specific morbidity, will incorporate the effect of any OD/OT. (To be clear, being diagnosed with a condition is not an outcome criterion.) Two, this strategy will simultaneously address any UD/UT, which will also be of major, if not more, importance to the person. Three, the weighting of these criteria by the person will overcome the preference insensitivity of the population rates of $\mathrm{OD} / \mathrm{OT}$ and complete the process of meeting the requirements of informed and preference-based consent.

\section{Conclusion}

Debates about OD/OT are useful, but in seeking to establish, classify and modify the sources of these phenomena located outside the individual decision, they represent a distraction from the central question: how do we enable, for the individual, the 'duallypersonalised' care that is optimal for them, i.e. combines their personalised preferences and individualised evidence into an evaluation of each option. Not only is any attempt to introduce $\mathrm{OD} / \mathrm{OT}$ rates as discrete information on a single criterion unlikely to be helpful, it also fails to address UD/UT simultaneously, or at all.

Fortunately, it is unnecessary to do so, given our vision of the future citizen empowered by MCDA-based DSTs. In these, the best available estimates of the performance rates of the available options on all-cause and condition-specific outcomes will implicitly incorporate group/population rates of OD/OT - and indeed of UD/UT as well.

We do not need to burden clinician and/or person with having to 'take into account and bear in mind' these phenomena. The problem at the individual level is not of possible over- or under-treatment, but of possible mis-treatment, i.e. management which is out of line with the optimal decision for the person, either because the relevant performance rates are not available, or are not drawn on, if available.

\section{References}

[1] Glasziou P, Jones MA, Pathirana T, Barratt AL, Bell KJL. Estimating the magnitude of Cancer Overdiagnosis in Australia. Med J Aust. 2020 212(4):163-68.

[2] Mesko B, Györffy Z. The rise of the empowered physician in the digital health era: Viewpoint. J Med Internet Res. 2019 21(3):e12490.

[3] Brodersen J. How to conduct research on overdiagnosis. Eur J Gen Pract 2017 23(1):78-82.

[4] Housten AJ, Lowenstein LM, Hoffman A, Jacobs LE, Zirari Z, Hoover DS, et al. A Review of the Presentation of Overdiagnosis in Cancer Screening Patient Decision Aids. MDM Policy Pract. 2019 4(2).

[5] Hersch J, Barratt A, Jansen J, Irwig L, McGeechan K, Jacklyn G, et al. Use of a decision aid including information on overdetection to support informed choice about breast cancer screening: A randomised controlled trial. Lancet. 2015 385(9978):1642-52. 